\title{
Biomedical research: a culture in crisis?
}

\author{
Eva L Feldman
}

Over the past 10 years, the scope of biomedical research in the US has widened, fueled by the substantial increase in the extramural research budget of the National Institutes of Health $(\mathrm{NIH})$. In parallel, the number of individuals at academic health centers participating in research has increased. There are indications, however, that the size and scope of the current enterprise might not be sustained.

This situation is illustrated by the recent meeting of the Peripheral Nerve Society (PNS), in July 2005. Although the PNS has a rich history of bringing together individuals focused on biomedical research and clinical investigation, the content of this year's meeting was largely clinical, as fewer abstracts were submitted from individuals engaged in basic biomedical research. How can this occur in the setting of the recent influx of resources for research?

One problem is that, despite increases in the research budget, the size of the present research enterprise is not sustainable at the current level of NIH funding, and faculty members at all levels are losing NIH support. In turn, academic health centers with faculty who depend on $\mathrm{NIH}$ funding are encountering increasing difficulty in funding the time required by faculty to perform high-quality biomedical research. This is further complicated by the clinical demands on faculty time and effort. Supplying neurological care provides an ongoing funding source, yet the required clinical time competes with the time needed to perform research. Although the long-term implications remain to be seen, early indicators, as simple as the scientific program of the PNS, or as measurable as the unprecedented number of vacancies in academic health centers, suggest that individuals are leaving the basic research enterprise. The allocation of increasingly scarce academic resources to
Effective

mentoring and

a change in

the academic

culture are

needed to

restore interest

in biomedical

research

careers

\section{EL Feldman is the \\ Russell N DeJong \\ Professor of Neurology at the University \\ of Michigan and an Advisory Board member of Nature Clinical Practice Neurology.}

\section{Competing interests \\ The author declares she has} no competing interests.

www.nature.com/clinicalpractice doi:10.1038/ncpneuro0053 provide faculty with protected time is essential to shape and ensure the future of the biomedical enterprise.

As the 'fuel' for the biomedical 'engine' is increasingly in short supply, several recent studies confirm that residents and fellows are losing interest in pursuing academic careers. The negative financial implications of electing an academic career are substantial, and private practitioners earn more than their counterparts in the same academic specialty. This can be a significant factor when the current median debt from a medical school education is in excess of US $\$ 100,000$. Furthermore, the 'holy grail' of professorship is not held in the same esteem by the current trainees as by their predecessors. These individuals seek a better work-life balance and more financial stability than do current senior faculty.

Effective mentoring and a change in the academic culture are needed to restore interest in biomedical research careers. Job sharing of biomedical research positions, greater access to recent technology, and the requirement of less 'face-to-face' time in the health center would all allow young physicians to integrate their personal lives into a meaningful professional life in biomedical research. The complexity of biomedical research also requires that individuals in academic health centers are mentored by a team of senior researchers. The model of one fellow working solely for the 'wise grey-haired' professor no longer provides the training required for success in the complex research enterprise. A forward-thinking program that provides a coherent team-based mentoring strategy, addressing the professional and family needs of the next generation of biomedical researchers, is mandatory.

The challenge to the academic community is clear and urgent. 